\title{
Research on High-Precision Attitude Control of Joint Actuator of Three-Axis Air-Bearing Test Bed
}

\author{
Zhiming Chen, Zhouhuai Luo $\mathbb{D}$, Yunhua Wu, Wei Xue, and Wenxing Li \\ College of Astronautics, Nanjing University of Aeronautics and Astronautics, Nanjing 210016, China \\ Correspondence should be addressed to Zhouhuai Luo; luozhouhuai@nuaa.edu.cn
}

Received 13 January 2021; Revised 27 February 2021; Accepted 11 March 2021; Published 26 March 2021

Academic Editor: Yongji Wang

Copyright ( 92021 Zhiming Chen et al. This is an open access article distributed under the Creative Commons Attribution License, which permits unrestricted use, distribution, and reproduction in any medium, provided the original work is properly cited.

\begin{abstract}
Three-axis air-bearing test bed is important semiphysical simulation equipment for spacecraft, which can simulate spacecraft attitude control, rendezvous, and docking with high confidence. When the three-axis air-bearing table is maneuvering at a large angle, if it is only controlled by the flywheel, it will cause the problems of slow maneuvering speed and high energy consumption, and when the external interference torque becomes large, the control accuracy will decline. A combined actuator including flywheel, air-conditioner thruster, and automatic balancing device is designed, and a hierarchical saturation PD control algorithm is proposed to improve the control accuracy and anti-interference ability of the three-axis air-bearing test bed. Finally, the mathematical simulation of the proposed control algorithm is carried out, and the physical verification is carried out on the threeaxis air-bearing test bed. The results show that the control algorithm has higher control accuracy than the traditional control algorithm, and the control accuracy is better than $0.1^{\circ}$ and basically meets the attitude control requirements of the ground simulation in-orbit satellite.
\end{abstract}

\section{Introduction}

With the continuous development of space technology, more and more satellites are used in space to perform different tasks [1]. Due to the high development cost, high maintenance cost, and difficult maintenance after entering orbit, it is very important to simulate and verify the satellite systems before entering space [2]. The three-axis air-bearing test bed is composed of an attitude measurement system, control system, air-bearing system, and other systems, which can effectively simulate the microgravity environment. We perform high-confidence simulations of tasks that need to be performed after the satellite enters space to ensure that the satellite completes the mission successfully. Among them, the interference torque has always been an important factor restricting the use of the three-axis air-bearing test bed. Airbearing test bed on the market is greatly affected by the interference torque when in use, and many scientific researchers only stay at reducing the interference torque experienced by the air-bearing test bed before using. Since the air-bearing test bed is a precision instrument, the interference torque of the milliN level will affect the control accuracy of the air-bearing test bed.

During use, factors such as ground vibration and airflow influence will increase the interference torque and affect the use of the air-bearing test bed. The purpose of this research is to use the joint actuator to reduce the interference torque of the three-axis air-bearing during the control process, improve the control accuracy, and perform simulation experiments such as high-confidence spacecraft attitude control. Many scientific research scholars have done a lot of research on the interference torque of the air-bearing test bed, but most of them are limited to analyzing the interference torque of the air bearing under the static state. Few people have studied the interference torque of the airbearing test bed in the control process [3-6]. Hua and Hao [7] designed a combined control algorithm for the flywheel and jet mechanism of the single-axis physical simulation test bed and carried out the simulation test. The simulation results show that the combined control can effectively shorten the maneuver time while maintaining high-precision control, aiming at the requirements of large-angle 
maneuver and high-precision control of Earth observation satellite.

Dong [8] proposed a joint actuator strategy based on thruster and flywheel, and the simulation results meet the design requirements. Haiying et al. [9] designed a combined control law of two kinds of biased momentum wheel and jet. The simulation results show that the control accuracy of the two methods is improved compared with the traditional scheme, and the steady-state control accuracy is up to $0.25^{\circ}$. Ling [10] designed the cold air thruster and reaction flywheel as the joint actuator of the small satellite, which realized the requirements of rapid maneuver and high-precision tracking of the small satellite. Saulnier and Perez Dand Gallardo [11] built a six-axis air-bearing test bed, which realized the automatic balance of the air-bearing test bed in the static state, but did not consider the interference torque generated by the air-bearing test bed movement. Jisu et al. [12] gave a detailed introduction to the principle and application of the full physical simulation of the satellite control system. Qilin et al. [13] conducted a theoretical analysis and research on the air-conditioning thrust of the five-degree-of-freedom air-bearing through the designed air-conditioning thrust test device, and the research results provided for the selection of suitable nozzles. The experimental basis was established, and it also laid the foundation for subsequent scholars to study the thrust optimization distribution plan. Dingjun et al. [14] obtained the basis for designing the nozzle by studying the relationship between the flow field pressure and the Mach number.

Recently, scientific researchers have proposed many new theories and methods for the control of nonlinear systems. Huo et al. [15] proposed a feasible decentralized eventtriggered control method under the framework of ACD (Adaptive Critical Design) for a class of constrained nonlinear systems. Chang et al. [16] studied an adaptive neural control method for a class of random switching nonlinear systems with actuator failure in the form of nonstrict feedback. The fault-tolerant control obtained by backstepping technology is designed to solve the problem of actuator failure.

Wang et al. [17] designed an adaptive control law based on the Lyapunov function, combined the backstepping method with the adaptive control law, and proposed an observer-based adaptive output feedback tracking control method, which can ensure that all the closed-loop system signals are bounded. Ma et al. [18] studied the problem of adaptive neural fault-tolerant control for a class of uncertain switched nonfeedback nonlinear systems with unmodeled dynamics and unmeasured states, by designing an adaptive fault-tolerant tracking controller to ensure that all closedloop signals are bounded under arbitrary switching. Xiong et al. [19] proposed a quantitative control strategy based on state feedback, which can be easily extended to the situation of static output feedback and effectively avoids some equation constraints in existing work. Li et al. [20] designed an adaptive fuzzy sliding mode controller and proposed an embedded attitude control system design for a $1 \mathrm{U}$ CubeSat and used three reaction flywheels as attitude actuators. Compared with the conventional proportional-integral- derivative controller, their controller has higher control accuracy. He et al. [21] investigated a relative coupling control strategy based on the sliding mode controller to realize the multiaxis servosynergic control with variable proportions during the operation of the system. Yuan et al. [22] designed a nonlinear disturbance observer and an adaptive backstepping sliding mode controller and then combined the two to design a hybrid control scheme.

The attitude actuator of the three-axis air-bearing table consists of cold air thruster, a reaction flywheel, and an automatic balance system. Most of the research done by domestic and foreign scholars focuses on the combination of cold air thrusters and reaction flywheels, and the combination of the three to form a joint executive agency is rare. To realize the rapid maneuver and high-precision control of the three-axis air-bearing test bed and to consider the influence of real-time disturbance torque and flywheel saturation, a joint actuator including flywheel, cold air thruster, and automatic balancing device is designed, and a hierarchical saturation PD control algorithm is proposed. Finally, the Simulink model and the real object of the three-axis airbearing test bed are built to simulate and verify the algorithm.

\section{General Scheme of the Control System for Three-Axis Air-Bearing Test Bed}

The control system of the three-axis air-bearing test bed is composed of an attitude sensor, attitude actuator, and integrated processing computer. Among them, the attitude sensor includes an inclinometer, gyroscope, and vision sensor, and the attitude actuator includes cold air thruster, a reaction flywheel, and an automatic balancing system. The control system is mainly used to ensure the stability of the three-axis air-bearing test bed and achieve the specified action.

2.1. Design of Flywheel System. There are two main working modes of reaction flywheel: speed mode and torque mode. In the speed mode, to achieve the command speed given by the controller, the flywheel outputs torque in the form of acceleration or deceleration for precise control. In torque mode, according to the command torque given by the controller, the flywheel adjusts the voltage and current to make the output electromagnetic torque equal to the command torque, to achieve the purpose of control. In this paper, the maximum speed of the reaction flywheel is $4500 \mathrm{r} / \mathrm{min}$ and the maximum output torque is $0.015 \mathrm{Nm}$, which is controlled by speed mode. The transfer function of the control signal and output torque is as follows [23]:

$$
G(s)=\underset{\zeta \subset(x, y, z)}{\operatorname{diag}}\left\{\frac{K_{\zeta} s}{s+K_{\zeta} s}\right\} .
$$

Among them, $G(s)$ represents the transfer function of the control signal and output torque in the speed mode of the reaction flywheel and $s$ represents the frequency domain,

$K_{\zeta}$ represents the torque coefficient of the internal motor of the reaction flywheel. The flywheel is installed in the form 
of three-orthogonal and one inclined installation. Four reaction flywheels are designed to be installed on the three-axis air-bearing test bed. The inclined flywheel is used as a spare flywheel to enhance the reliability of the three-axis airbearing test bed. The actual installation of the flywheel is shown in Figures 1 and 2.

2.2. Design of Cold Air Thrust System. The cold air thruster can provide the moment of force to realize the rapid maneuvering of the three-axis air-bearing test bed. In this paper, the high-pressure gas in the cylinder is reduced to $0.5 \mathrm{Mpathrough}$ the pressure reducing valve, and the thrust provided by the reduced gas through the Laval nozzle is about $0.2 \mathrm{~N}$. This article first introduces the PWM wave and then controls the opening time of the solenoid valve to obtain the desired torque. The opening time of the thruster in a control cycle is

$$
t_{\text {on }}= \begin{cases}T, & \frac{\tau u_{i}}{F_{i}} \geq T, \\ \frac{\tau u_{i}}{F_{i}}, & \tau \leq \frac{\tau u_{i}}{F_{i}}<T, \\ 0, & \frac{\tau u_{i}}{F_{i}}<\tau .\end{cases}
$$

Among them, $T=0.2 \mathrm{~s}$ is the control cycle, $\tau=10 \mathrm{~ms}$ is the time required for the switching response of the solenoid valve, $u_{i}$ is the command thrust of the $i$ cold air thruster, and $F_{i} \approx 0.2 \mathrm{~N}$ is the thrust that can be provided when the cold air thruster is fully injected.

\subsection{Design of Dynamic Disturbance Torque Compensation} Mechanism. Due to the limited machining accuracy of the three-axis air-bearing test bed, it is difficult to achieve the complete average distribution of mass and the stiffness in all directions. In the actual control process, due to the continuous consumption of cold air volume in the gas cylinder of the air-bearing test bed and the influence of external airflow and vibration, the original leveling state is broken, and the center of mass deviates from the center of rotation. It is necessary to compensate for the dynamic disturbance torque in real time to make the rotation center of the airbearing test bed coincide with the mass center and make the eccentricity $r=0 \mathrm{~m}$. The dynamic disturbance torque compensation mechanism designed in this paper, namely, automatic balancing device, is composed of a high-precision stepping motor, mass block, and screw rod, as shown in Figure 3.

Firstly, according to the principle of error attitude angle and right-hand rule, the direction of dynamic disturbance torque is estimated in real time; at the same time, the magnitude of dynamic disturbance torque is estimated through the actual output torque of the flywheel; finally, the dynamic disturbance torque is compensated by rotating the high-precision stepping motor and moving the mass on the automatic balancing device in real time. Taking the target

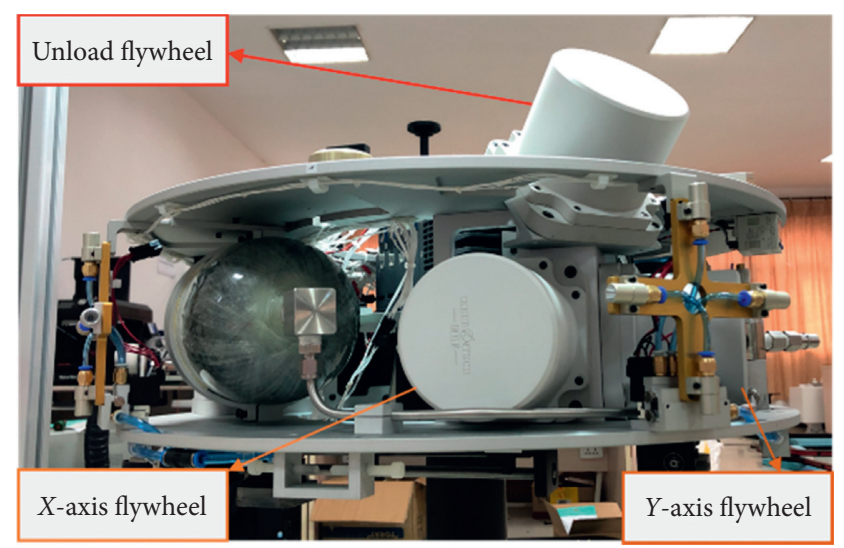

Figure 1: $X$-axis, $Y$-axis, and oblique reaction flywheel.

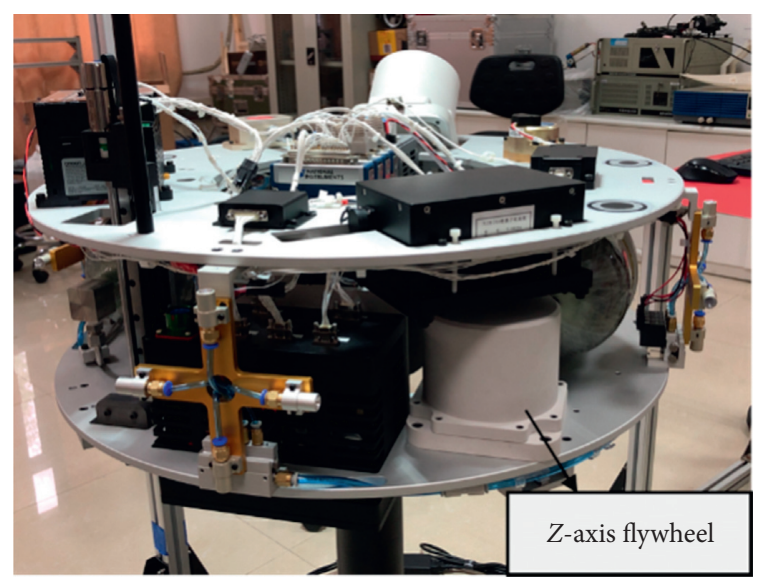

FIgURE 2: $Z$-axis reaction flywheel.

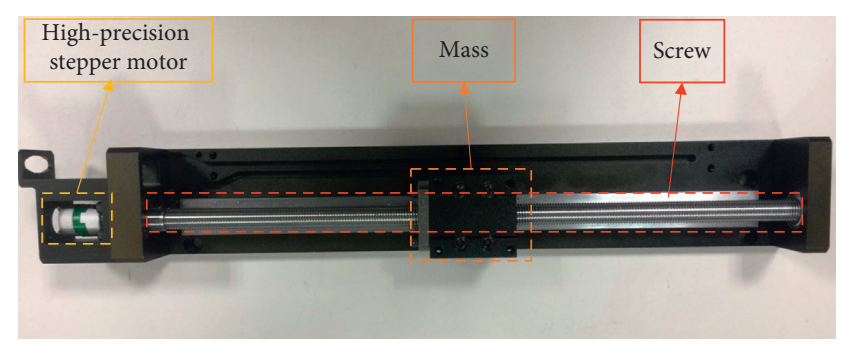

FIgURe 3: Dynamic disturbance torque compensation mechanism.

attitude angle $\left[\begin{array}{lll}0^{\circ} & 0^{\circ} & 0^{\circ}\end{array}\right]$, but due to the influence of dynamic disturbance torque, the air-bearing test bed can only be controlled $\left[\begin{array}{lll}0.2^{\circ} & 0^{\circ} & 0^{\circ}\end{array}\right]$ in practice as an example.

As shown in Figure 4, according to the principle of the right-hand rule, the positive direction of the $Y$-axis is heavier, $r_{y}>0$. Therefore, the automatic balancing device in the $Y$-axis direction is selected, and the high-precision stepping motor rotates according to the control command, driving the mass block to move in the $Y$-negative direction along the screw rod, to compensate for the dynamic interference torque in real time. In the same way, other compensation strategies can be obtained. If the target attitude angle is $\alpha$ and the actual attitude angle is $\beta$, the error 


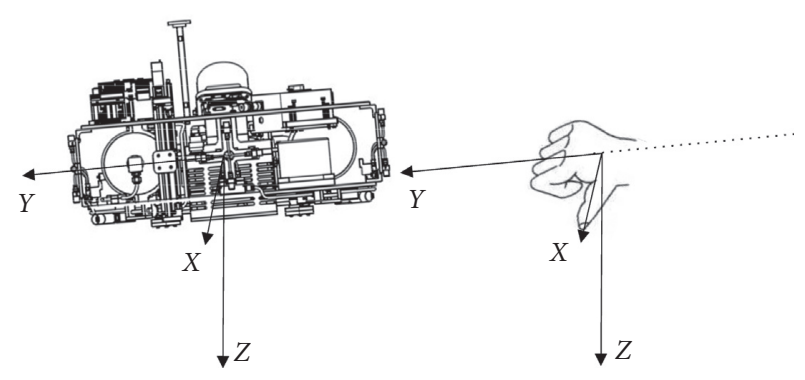

FIGURE 4: Judging the direction of eccentricity by the right-hand rule.

attitude angle is $\beta-\alpha=\delta$. The relationship between the error attitude angle and the moving direction of the mass is shown in Table 1.

\section{Design of Attitude Control Algorithm for Three-Axis Air-Bearing Test Bed}

\subsection{Scheme Design of Joint Execution Agency}

(1) Design of combined actuator of cold air thruster and flywheel: to shorten the overcontrol time and ensure high-precision control, the maximum angular velocity of the air-bearing test bed is automatically adjusted according to the error attitude angle $\delta$, and the control process of the air-bearing test bed is divided into three stages.

(i) The first stage is the rapid maneuvering stage; when $|\delta| \geq 1^{\circ}$, the controller automatically sets the maximum angular velocity of the airbearing test bed to $3 \mathrm{deg} / \mathrm{s}$, which is controlled by the cold air thruster.

(ii) The second stage is approaching the target attitude stage; when $0.5^{\circ} \leq|\delta|<1^{\circ}$, the maximum angular velocity is automatically set to $1 \mathrm{deg} / \mathrm{s}$. Due to the inertia effect, the actual angular velocity of the air-bearing test bed decreases slowly. To avoid flywheel torque saturation, the flywheel and the cold air thruster are controlled at the same time in this stage.

(iii) The third stage is the high-precision control stage of the air-bearing test bed, the maximum angular velocity is automatically set to $0.5 \mathrm{deg} / \mathrm{s}$, the control precision limit of the cold air thruster, and carries out high-precision control through the flywheel. To avoid the problem of flywheel speed saturation in the control process, a method is designed to unload the flywheel by cold air thruster when the absolute value of flywheel speed is greater than or equal to and stop unloading when the absolute value of flywheel speed is less than $500 \mathrm{r} / \mathrm{min}$.

(2) Design of combined actuator of cold air thruster and flywheel: after the first and second stage control of the three-axis air-bearing test bed, the amount of
TABLE 1: Relationship between error attitude angle and mass moving direction.

\begin{tabular}{lccc}
\hline Axis & $\delta$ & $\begin{array}{c}\text { Automatic balancing } \\
\text { device }\end{array}$ & $\begin{array}{c}\text { Mass movement } \\
\text { direction }\end{array}$ \\
\hline $\begin{array}{l}X- \\
\text { axis }\end{array}$ & $>0$ & $Y$-axis & $Y$-axis negative direction \\
$X-$ \\
$\begin{array}{l}\text { axis } \\
Y-\end{array}$ & $<0$ & $Y$-axis & $Y$-axis positive direction \\
$\begin{array}{l}\text { axis } \\
Y-\end{array}$ & $>0$ & $X$-axis & $X$-axis positive direction \\
axis & $<0$ & $X$-axis & $X$-axis negative direction \\
\hline
\end{tabular}

cold air in the cylinder is reduced. In the actual control process, it is also vulnerable to the interference of external airflow and vibration. The rotation center of the air-bearing test bed will deviate slightly from the center of mass, which will produce real-time dynamic interference torque and affect the control accuracy of the reaction flywheel. In the third stage of the process, according to the error attitude angle and the principle of the right-hand rule, the direction of the dynamic interference torque is judged, and the dynamic interference torque is estimated according to the actual output torque of the reaction wheel, and the real-time compensation is carried out by the automatic balancing device. The working principle of the joint actuator designed in this paper is shown in Figure 5.

3.2. Kinematics and Dynamics Equations of Air-Bearing Test Bed. Taking the camera coordinate system of the vision measurement system as the reference system, when the three-axis air-bearing test bed is maneuvering, the body coordinate system rotates with the air-bearing test bed, the maneuvering angular velocity of the air-bearing test bed can be expressed as $\omega=\left[\begin{array}{lll}\omega_{x} & \omega_{y} & \omega_{z}\end{array}\right]^{T}$, and the attitude quaternion can be expressed as $q=\left[q_{0}, q\right]^{T}$, where $q$ is the vector part of quaternion and $q=\left[q_{1}, q_{2}, q_{3}\right]^{T} . q_{0}$ is the scalar part of the quaternion, and the attitude kinematics equation of satellite-based on quaternion can be obtained as follows [24]:

$$
\begin{aligned}
\dot{q} & =\left[\begin{array}{c}
\dot{q}_{0} \\
\dot{q}
\end{array}\right]=\left[\begin{array}{c}
-\frac{1}{2} q^{T} \omega \\
\frac{1}{2} T\left(q_{0}, q\right) \omega
\end{array}\right], \\
T\left(q_{0}, q\right) & =q_{0} I_{3 \times 3}+q[\times],
\end{aligned}
$$

where $I_{3 \times 3}$ is the identity matrix and $q[x]$ is the antisymmetric cross-product matrix:

$$
[q \times]=\left[\begin{array}{ccc}
0 & -q_{3} & q_{2} \\
q_{3} & 0 & -q_{1} \\
-q_{2} & q_{1} & 0
\end{array}\right] .
$$




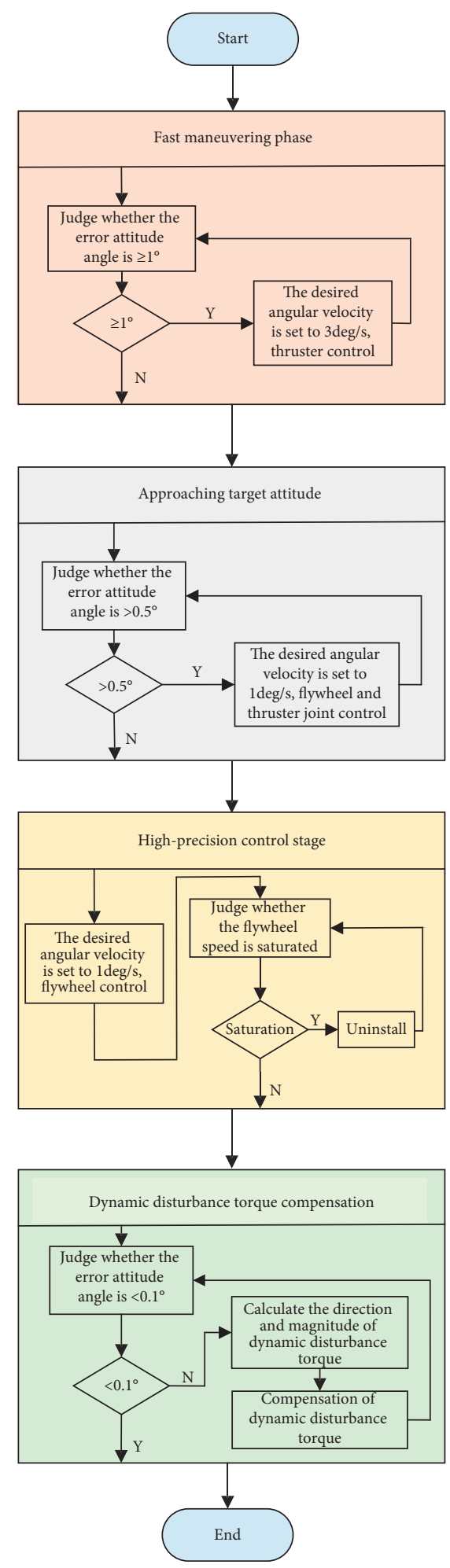

FIGURE 5: Working principle of joint actuator.

In this paper, the three-axis air-bearing test bed is idealized as a rigid body model, and its attitude dynamic model can be expressed as a rigid body model [25]:

$$
\dot{\omega}=J_{A}^{-1}\left(-\omega[\times] J_{A} \omega+T_{k}+T_{d}\right),
$$

where $\mathrm{T}_{k}$ is the control torque, $\mathrm{T}_{d}$ is the interference torque, and $\mathrm{J}_{A}$ is the moment of inertia of the air-bearing test bed.
3.3. Modeling of Cold Air Thrust Distribution. In the fast maneuvering stage of the air-bearing test bed, the cold air thruster is controlled as the actuator. To save the cold air volume of the cylinder, the thrust is distributed by the pseudoinverse method. The mathematical model of thrust distribution is as follows:

$$
C=D F,
$$

where $C$ is the control instruction given by the control law. $D$ is the $m \times n$ dimensional thruster configuration matrix, and the universal function is

$$
J(F)=\sum_{i=1}^{n} F_{i}^{2}=F^{T} F,
$$

where $F$ is the thrust array composed of all thrusters, which can be obtained by combining with the mathematical model of thrust distribution:

$$
F=D^{T}\left(D D^{T}\right)^{-1} C,
$$

where $D^{T}\left(D D^{T}\right)^{-1}$ is the pseudoinverse of $D$. To ensure $F>0$, the initial solutions obtained by the pseudoinverse method are grouped according to the positive and negative thrust:

$$
F=\left[\begin{array}{l}
F_{n} \\
F_{p}
\end{array}\right], \quad F_{n}<0, F_{p}>0,
$$

where $F_{n}$ is the negative thrust and $F_{p}$ is the positive thrust; at the same time, the efficiency matrix is grouped correspondingly [17]:

$$
D=\left[\begin{array}{l}
D_{n} \\
D_{p}
\end{array}\right],
$$

where $D_{n}$ is the negative efficiency and $D_{p}$ is the positive efficiency, and the thrust of the negative group is set to zero:

$$
u_{1}=\left[\begin{array}{lll}
0 & \cdots & 0
\end{array}\right] \text {, }
$$

where $u_{1}$ is a zero matrix and the expected quantity after the allocation is redistributed:

$$
M_{d}=D_{p} u_{2}
$$

where $u_{2}$ is the final corrected thrust without negative value and $M_{d}$ is the expected torque [26-29].

3.4. Hierarchical Saturated PD Control Law. Considering the limited factors such as flywheel saturation, a hierarchical saturation PD control algorithm is designed, which is controlled by air-conditioner and flywheel with high precision.

The general form of traditional PD control law for threeaxis air-bearing test bed is as follows:

$$
T=-K q_{e}-D \omega+[\omega \times] J_{A} \omega,
$$

where $q_{e}$ is the vector part of the error quaternion and $K$ and Dare the control parameters. Infinite norm saturation function is introduced to the traditional PD control law: 


$$
\operatorname{sat}_{x_{\max }}(x)= \begin{cases}x, & \sigma(x) \leq x_{\max } \\ x \frac{x_{\max }}{\sigma(x)}, & \sigma(x)>x_{\max }\end{cases}
$$

By introducing $q_{e}$ into the infinite norm saturation function, we can get the following results:

$$
\operatorname{sat}_{\sigma}\left(q_{e}\right)= \begin{cases}q_{e}, & \left\|q_{e}\right\|_{\infty} \leq q_{e \max }, \\ q_{e} \frac{q_{e \max }}{\left\|q_{e}\right\|_{\infty}}, & \left\|q_{e}\right\|_{\infty}>q_{e \max } .\end{cases}
$$

When the maximum output torque of the actuator is $\lambda$, the limiting conditions of the control force are as follows:

$$
\text { sat }_{\sigma}(\lambda)= \begin{cases}\lambda, & \|\lambda\|_{\infty} \leq \lambda_{\max }, \\ \lambda \frac{\lambda_{\max }}{\|\lambda\|_{\infty}}, & \|\lambda\|_{\infty}>\lambda_{\max } .\end{cases}
$$

Thus, the PD control law of the joint actuator of the three-axis air-bearing test bed is obtained:

$$
T=\operatorname{sat}_{\sigma}\left[-K_{s a t}\left(P q_{e}\right)-D \omega+\omega[\times] J_{A} \omega\right] .
$$

Here, for the convenience of design, we take $P$ as the unit diagonal matrix, and KandDare as follows [30]:

$$
\begin{aligned}
& K=\operatorname{diag}\left(\left[k_{1}, k_{2}, k_{3}\right]\right) \cdot J_{A}, \\
& D=\operatorname{diag}\left(\left[d_{1}, d_{2}, d_{3}\right]\right) \cdot J_{A} .
\end{aligned}
$$

Therefore, (17) can be simplified as

$$
T=\operatorname{sat}_{\sigma}\left[-k J_{A} s a t_{\sigma}\left(q_{e}\right)-d J_{A} \omega+\omega[\times] J_{A} \omega\right] .
$$

Finally, according to the design scheme of the joint actuator, the error attitude angle and the command torque in the current state are judged, and the cold air thruster or flywheel is selected to execute the command torque.

\subsection{Modeling and Algorithm Design of Dynamic Disturbance} Torque Compensation Mechanism. Three dynamic disturbance torque compensation mechanisms, namely, automatic balancing devices, are installed on three coordinate axes in the form of orthogonal distribution. The unit vectors of the mass blocks on the three automatic balancing devices in the frame coordinate system of the air-bearing test bed are represented by $\varepsilon_{1}, \varepsilon_{2}$, and $\varepsilon_{3}$, respectively.

Assuming that the center of mass of the air-bearing test bed deviates from the center of rotation under the interference, the position vectors of the three mass blocks relative to the frame coordinate system are $\boldsymbol{\sigma}=\left[\begin{array}{lll}\sigma_{1} & \sigma_{2} & \sigma_{3}\end{array}\right]$ and move under the control command $l_{i}=\left[\begin{array}{lll}l_{1} & l_{2} & l_{3}\end{array}\right]$. When the center of mass coincides with the center of rotation again, the mass position vector is $\partial_{i}$.

$$
\begin{aligned}
& \partial_{1}=\sigma_{1}+l_{1} \varepsilon_{1}, \\
& \partial_{2}=\sigma_{2}+l_{2} \varepsilon_{2}, \\
& \partial_{3}=\sigma_{3}+l_{3} \varepsilon_{3} .
\end{aligned}
$$

In this case, the center of mass of the three-axis airbearing test bed can be expressed as

$$
r_{a}=\frac{1}{m} \int_{B} \partial d m=\frac{1}{m}\left[\left(m-\sum_{i=1}^{3} m_{i}\right) \partial_{0}+\sum_{i=1}^{3} m_{i} \partial_{i}\right],
$$

where $m$ is the total mass of the floating part of the airbearing test bed (including mass blocks), $m_{1}, m_{2}$, and $m_{3}$ are the mass blocks of the three automatic balancing devices, respectively, and $\partial_{0}$ is the mass center of the air-bearing test bed without mass blocks. When the three mass blocks move $\triangle \mathrm{l}$ under the control command, the mass center position of the air-bearing test bed is

$$
r_{b}=\frac{1}{m}\left[\left(m-\sum_{i=1}^{3} m_{i}\right) \partial_{0}+\sum_{i=1}^{3} m_{i}\left(\sigma_{i}+\left(l_{i}+\Delta l_{i}\right) \varepsilon_{i}\right)\right] .
$$

By subtracting formula (21) from formula (22), we can get the following formula:

$$
\Delta r=\frac{1}{m} \sum_{i=1}^{3} m_{i} \Delta l_{i} \varepsilon_{i}
$$

Therefore, the relationship between the mass moving distance and the mass center moving of the air-bearing test bed can be obtained:

$$
\Delta l_{i}=-\frac{m}{m_{i}} r_{i}
$$

The precision of a high-precision stepping motor is $1 / 3000$ turns, and the mass block can move $0.01 \mathrm{~m}$ for each turn, so the minimum step of mass block moving is $1 / 300000 \mathrm{~m}$. The relationship among the number of turns $k$, eccentricity $r_{i}$ and mass block moving amount $l_{i}$ of stepper motor is as follows:

$$
100 k=l_{i}=-\frac{m}{m_{i}} r_{i}
$$

If the dynamic disturbance torque is defined as $M_{c}$, the actual output torque of flywheel is $M_{r}$, and the angle of the floating part of air-bearing test bed relative to the horizontal plane is $\xi_{i}(i=x, y)$, then

$$
M_{c} \approx M_{c}=m g r_{i} \sin \xi_{i} \text {. }
$$

Therefore, the dynamic disturbance torque $M_{c}$ is estimated by the actual output torque of the flywheel, and the tilt angle $\xi_{i}$ of the air-bearing test bed is measured by the attitude measurement system of the air-bearing test bed. Then the eccentricity $r_{i}$ can be calculated, and the number of turns that the high-precision motor needs to rotate can be calculated. 


\section{Simulation Results and Analysis}

4.1. Simulation of Initial Value Settings and Results. To show the control effect more intuitively, this paper first sets up the control situation under the influence of dynamic disturbance torque in the simulation part, then compensates the dynamic disturbance torque in real time, and compares the two. Moment of inertia of three-axis air-bearing test bed is

$$
J_{A}=\left[\begin{array}{ccc}
0.878 & 0.044 & 0.023 \\
-0.044 & 0.962 & 0.032 \\
-0.023 & -0.032 & 1.474
\end{array}\right] \mathrm{kg} \cdot \mathrm{m}^{2} \text {. }
$$

The mass of the floating part of the three-axis air-bearing test bed is $m=30.6 \mathrm{~kg}$, the maximum output torque of the flywheel is $0.015 \mathrm{Nm}$, the maximum output torque of the thruster is $0.06 \mathrm{Nm}$, the mass of the mass block is $m_{i}=0.4 \mathrm{~kg}$, and the control parameters Kand Dare 1.85.

Set the initial attitude angle as $\left[\begin{array}{lll}0^{\circ} & 0^{\circ} & 0^{\circ}\end{array}\right]$ and the target attitude angle as $\left[\begin{array}{lll}0^{\circ} & 10^{\circ} & 0^{\circ}\end{array}\right]$ during the maneuvering process of the air-bearing test bed, due to the constant consumption of cold air in the cylinder, external airflow, vibration, and other interference reasons, the center of mass and the rotation center no longer coincide, and the original state of leveling is broken.

Set the interference torque of air-bearing test bed to

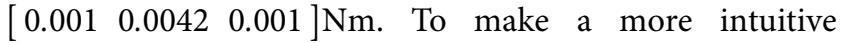
comparison, the $Y$-axis interference torque is compensated at $250 \mathrm{~s}$. To make a more intuitive comparison, the interference torque of the $Y$-axis is compensated at $250 \mathrm{~s}$. The simulation results are shown in Figures 6-8.

4.2. Simulation Analysis. From the simulation results in Figures 6-8, it can be seen that, in $0-17.8 \mathrm{~s}$, the $Y$-axis error attitude angle is greater than $1^{\circ}$, and it is in the rapid maneuvering stage, and the air-bearing table makes a largeangle maneuver under the action of the cold air thruster. In $18-19.4 \mathrm{~s}$, the $Y$-axis error attitude angle is between $0.5^{\circ}$ and $1^{\circ}$, and at the stage of approaching the target attitude, the flywheel and the cold air thruster are jointly controlled;

In 19.6 - 250s, the $Y$-axis error attitude angle is between $0.1^{\circ}$ and $0.5^{\circ}$. It is in the high-precision control stage, and the flywheel is used for high-precision control. However, due to the excessively large dynamic interference torque, the requirement that the control accuracy is better $0.1^{\circ}$ cannot be met at this time.

During this period, the speed of the flywheel was greater than $1500 \mathrm{r} / \mathrm{min}$. The cold air thruster was used to unload the flywheel to ensure that the actual speed of the flywheel was always within the preset range. In250s, the dynamic disturbance torque is compensated. In the following $250.2 \mathrm{~s}$ to400s, under the control of the flywheel, the control accuracy is always better than $0.5^{\circ}$.

\section{Analysis of Experiment and Test Results}

5.1. Experimental Initial Value Settings and Results. The initial value of the experimental part is the same as the initial

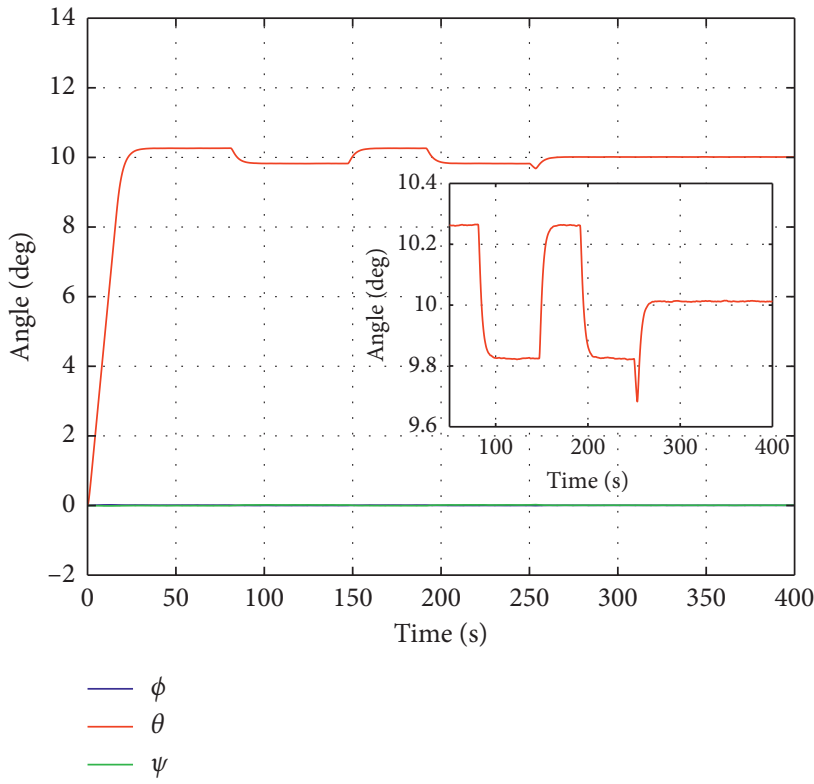

Figure 6: Attitude angle of air-bearing test bed.

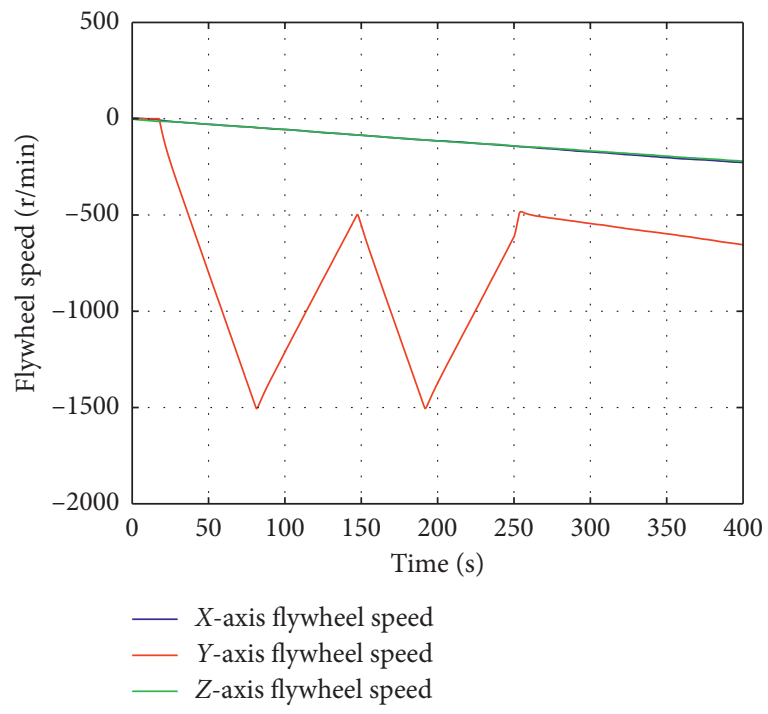

FIgURE 7: Flywheel speed.

value set in the simulation part, and the center of rotation of the air-bearing table coincides with the center of mass at the initial moment.

The target attitude angle command is shown in Figure 9. The target attitude angles of the air-bearing test bed are set to $\left[\begin{array}{lll}0^{\circ} & 0^{\circ} & 0^{\circ}\end{array}\right]\left[\begin{array}{lll}10^{\circ} & 0^{\circ} & 0^{\circ}\end{array}\right],\left[\begin{array}{lll}12^{\circ} & 0^{\circ} & 0^{\circ}\end{array}\right]$, $\left[\begin{array}{lll}0^{\circ} & 0^{\circ} & 0^{\circ}\end{array}\right]$, and $\left[\begin{array}{lll}-12^{\circ} & 0^{\circ} & 0^{\circ}\end{array}\right]$ in periods of $0-195 \mathrm{~s}$, $195.2-250 \mathrm{~s}, 250.2-265 \mathrm{~s}, 265.2-307 \mathrm{~s}$, and $307.2-400 \mathrm{~s}$. The experimental test results are shown in Figures 10-12.

5.2. Experimental Results and Analysis. It can be seen from the experimental results in Figures 9-11 that the actual attitude angle of the air-bearing test bed is highly consistent with the target attitude angle. At the initial time, because the 


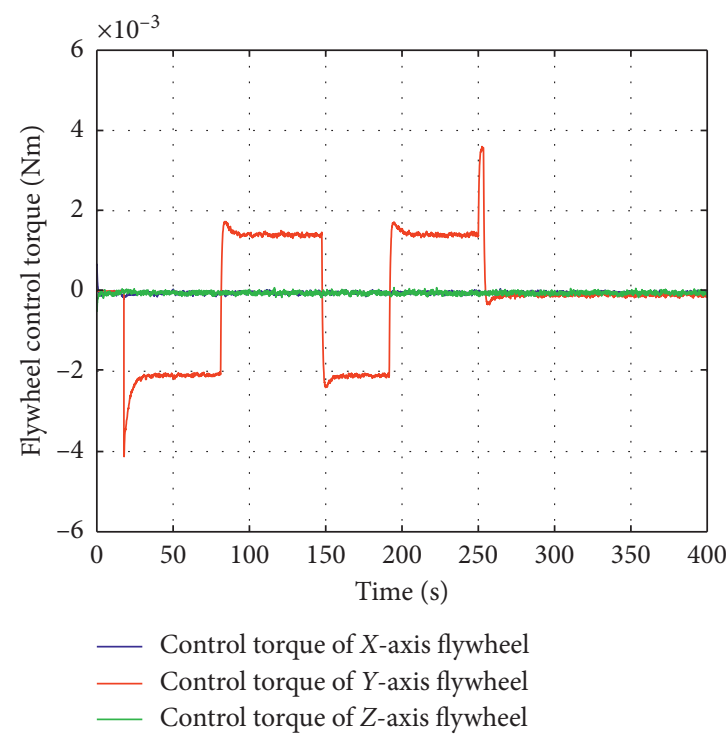

Figure 8: Flywheel control torque.

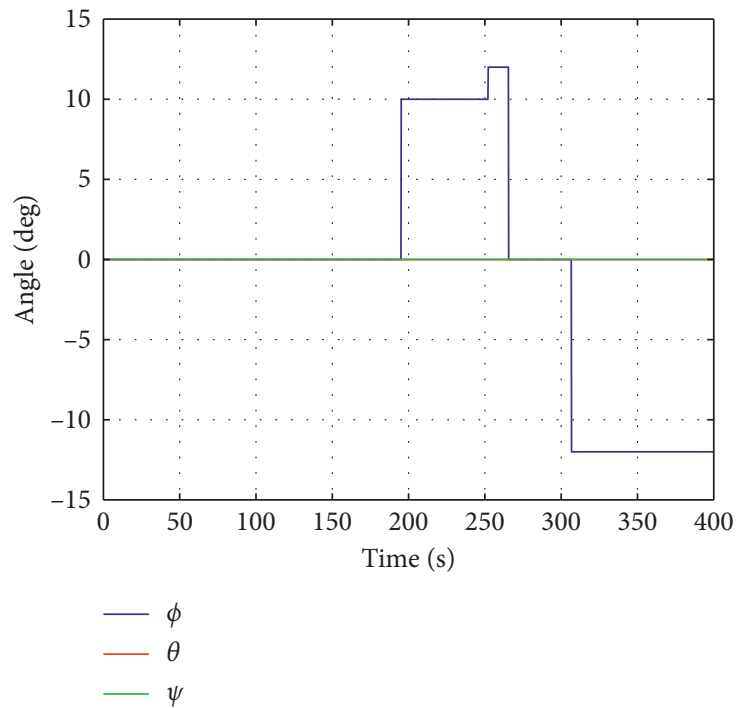

Figure 9: Angle of target attitude.

rotation center of the air-bearing test bed coincides with the mass center, the control accuracy is better than $0.1^{\circ}$ in $0-195 \mathrm{~s}$. In $195-250 \mathrm{~s}$, the target attitude angle is $\left[\begin{array}{lll}10^{\circ} & 0^{\circ} & 0^{\circ}\end{array}\right]$, and within $195.2-209.2 \mathrm{~s}$, the axis error attitude angle of the air-bearing test bed is greater than $3^{\circ}$. It is in the rapid maneuvering stage, and the air-bearing test bed makes large-angle maneuvers under the action of the cold air thruster. In $209.4-210.2 \mathrm{~s}$, the $Y$-axis error attitude angle is between $0.5^{\circ}$ and $1^{\circ}$. It is in the approaching target attitude stage, and the flywheel and the cold air thruster are jointly controlled. In $210.4-225 \mathrm{~s}$, the $Y$-axis error attitude angle is between $0.1^{\circ}$ and $0.5^{\circ}$, which is in the high-precision control stage.

However, due to the existence of dynamic disturbance torque, the control accuracy of the air-bearing table cannot reach the requirement of better than $0.1^{\circ}$. At this time, judging by the right-hand rule, there is a dynamic interference torque along the negative direction of the $Y$-axis, and the actual output torque of the flywheel shows that the dynamic interference torque is about $0.015 \mathrm{Nm}$.

At 225s, after moving the mass of the $Y$-axis automatic balancing device to the positive direction of the $Y$-axis, the interference torque is compensated in real time, and the control accuracy is improved and is better than $0.1^{\circ}$. After that, the air-bearing table maneuvered according to the target attitude commands in each period time. It can be seen that although the air-bearing table moves around the $X$-axis slightly, the other two axes are slightly affected, but the control accuracy after stability is better $0.1^{\circ}$. 


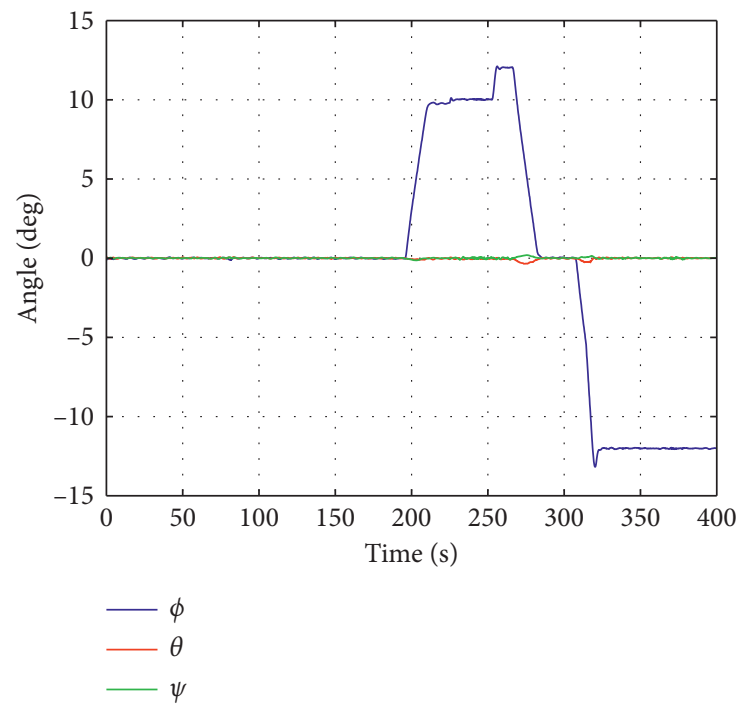

Figure 10: Actual attitude angle.
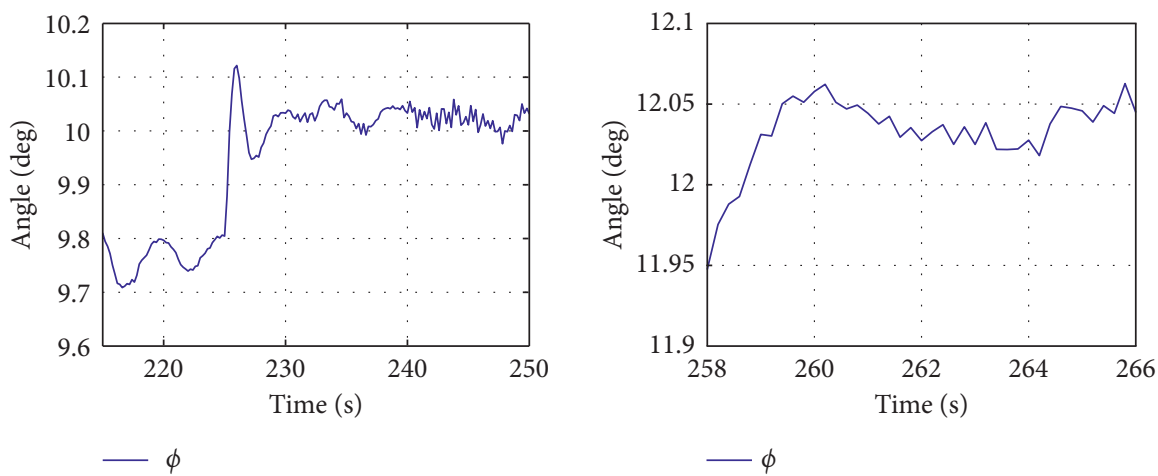

(a)

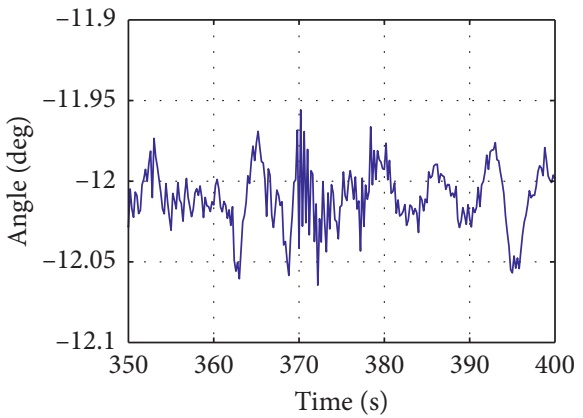

(b)

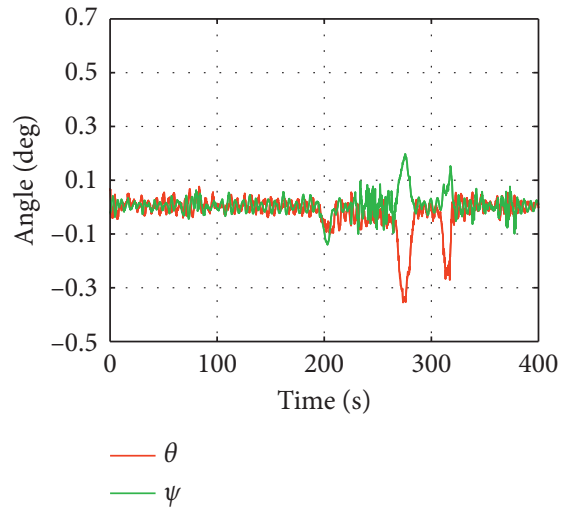

(c)

(d)

Figure 11: Local amplification of actual attitude angle. 


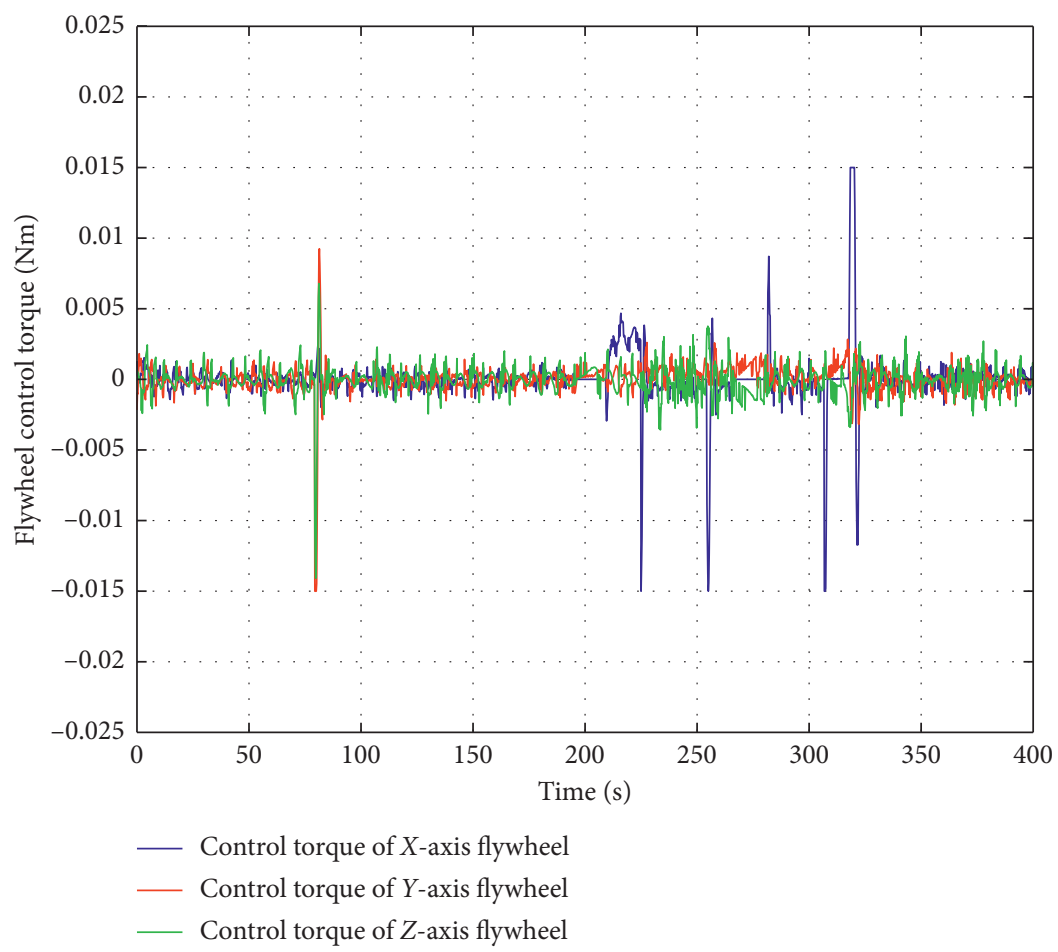

Figure 12: Flywheel control torque.

\section{Concluding Remarks}

This article is aiming at the three-axis air-bearing test bed when maneuvering at a large angle; if relying solely on flywheel control, it will cause long control time and high energy consumption. And in the control process, due to factors such as cold air consumption and external interference, the original leveling state of the air-bearing table is broken, which affects the control accuracy of the air-bearing test bed. In this paper, a joint actuator including flywheel, thruster, and automatic balancing device is designed.

The overall control process of the air-bearing table is subdivided into three stages to shorten the overall control time and reduce energy consumption. The direction of the interference torque is judged in real time by the right-hand rule principle and the error attitude angle, the dynamic interference torque is estimated according to the actual output torque of the flywheel, and the mass block on the automatic balance device is moved in real time to compensate for the dynamic interference torque in real time, to realize the three-axis air-bearing large-angle maneuvering and high-precision control and also to improve the antiinterference ability of the air-bearing test bed.

Due to time reasons, this article uses the hierarchical saturated PD control algorithm when studying the control algorithm and has not conducted in-depth research on other control algorithms. In future research work, it is planned to study a variety of advanced control algorithms and verification by three-axis air-bearing test bed.

\section{Data Availability}

The simulation data of this work were obtained from the Simulink model in MATLAB, and the experimental data were obtained from experimental tests.

\section{Conflicts of Interest}

The authors declare that they have no conflicts of interest in connection with the work submitted.

\section{References}

[1] Y. Shirui, "Weaving China space TT and C network," Space Exploration, vol. 35, no. 9, pp. 17-30, 2020.

[2] W. Yousong, Research on Satellite Attitude Control and Ground Simulation, Harbin Institute of Technology, Harbin, China, 2014.

[3] X. Dong, Research on Balancing System of Three-axis Air Bearing Test-Bed, Harbin Institute of Technology, Harbin, China, 2009.

[4] Y. W. Yanbing and Tong, "Analysis of unbalanced moment caused by gravity gradient of three-axis air bearing test-bed," Machine Tools and Hydraulics, vol. 45, no. 1, pp. 40-42, 2017.

[5] N. Brij, "Automatic mass balancing of air-bearing-based three-Axis rotational spacecraft simulator," Journal of Guidance, Control, and Dynamics, vol. 32, no. 3, pp. 10071017, 2009.

[6] S. Yeonchoi, Automatic Mass Balancing System for the 5-DOF Spacecraft Simulator, Daniel Guggenheim School of Aerospace Engineering, Denmark, UK, 2016. 
[7] G. Hua and Z. Hao, "Research on physical simulation technology of joint control for large-angle maneuver of aircraft attitude," Computer Measurement and Control, vol. 18, no. 10, pp. 2315-2317, 2010.

[8] Y. Dong, Research on Fast Attitude Maneuver and Stability Control Method of Agile Satellite, Harbin Institute of Technology, Harbin, China, 2009.

[9] L. Haiying, H. Wand, and C. Zhiming, "Combined control for three-axis stabilization of micro-satellite using thrusters and single pitch bias momentum wheel," Journal of System Simulation, vol. 21, no. 7, pp. 2023-2026, 2009.

[10] C. Ling, A Study Of Micro-satellite High-Precision Attitude Control Algorithm And Build Air Bearing Test-Bed, Nanjing University of Aeronautics and Astronautics, Nanjing, China, 2018.

[11] K. Saulnier and D. Perez Dand Gallardo, "A six-degree-of -freedom hardware-in-the-loop simulator for small spacecraft," ActaAstronautica, vol. 105, no. 12, pp. 444-462, 2014.

[12] L. Jisu, M. Xiaogang, and Z. Jinjiang, "Design of thrust allocation algorithm for three degrees of freedom air bearing test-bed," Aerospace Control, vol. 22, no. 2, pp. 37-45, 2004.

[13] Y. Qilin, X. Jiang, and Y. Qingjun, "System design cold air thrust test device for air flotation table," Machine Tool and Hydraulics, vol. 9, 2009.

[14] W. DingJun, S. Huiling, and B. Shaoqing, "Simulation and analysis of flow field in the throttle port of pressure reducing valve," Rocket Propulsion, vol. 35, no. 6, pp. 37-40, 2009.

[15] X. Huo, H. R. Karimi, X. Zhao et al., “Adaptive-critic design for decentralized event-triggered control of constrained nonlinear interconnected systems within an identifier-critic framework," IEEE Transactions On Cybernetics, pp. 41-54, 2021.

[16] Y. Chang, S. Zhang, N. D. Alotaibi et al., "Observer-based adaptive finite-time tracking control for a class of switched nonlinear systems with unmodeled dynamics," IEEE Access, vol. 8, pp. 4782-4790, 2020.

[17] Y. Wang, B. Niu, H. Wang et al., "Neural network-based adaptive tracking control for switched nonlinear systems with prescribed performance: an average dwell time switching approach," Neurocomputing, vol. 435, pp. 295-306, 2021.

[18] L. Ma, N. Xu, X. Zhao, G. Zong, and X. Huo, "Small-gain technique-based adaptive neural output-feedback faulttolerant control of switched nonlinear systems with unmodeled dynamics," IEEE Transactions on Systems, Man, and Cybernetics: Systems, vol. 49, pp. 1-12, 2020.

[19] J. Xiong, X. Chang, J. H. Park et al., "Nonfragile fault-tolerant control of suspension systems subject to input quantization and actuator fault," International Journal of Robust and Nonlinear Control, vol. 30, pp. 1-24, 2020.

[20] J. Li, M. Post, T. Wright, and R. Lee, "Design of attitude control systems for CubeSat-class nanosatellite," Journal of Control Science and Engineering, vol. 2013, Article ID 657182, 15 pages, 2013.

[21] J. He, X. Jiang, C. Zhang et al., "Multiaxis servo synergic control based on sliding mode controller," Journal of Control Science and Engineering, vol. 2019, Article ID 9249270, 16 pages, 2019.

[22] W. Yuan, G. Gao, and J. Li, "Adaptive backstepping sliding mode control of the hybrid conveying mechanism with mismatched disturbances via nonlinear disturbance observers," Journal of Control Science and Engineering, vol. 2020, Article ID 7376503, 13 pages, 2020.
[23] W. Kai, Research on Satellite Fast Attitude Maneuver Technology Based on Flywheel and Thruster Joint Control, Harbin Institute of Technology, Harbin, China, 2012.

[24] P. JunFan, Research on Attitude Control Simulation System of Small Three-axis Air Bearing Test-Bed for Agile Microsatellite, Nanjing University of Aeronautics and Astronautics, Nanjing, China, 2017.

[25] Z. Zhongzheng, Research on Satellite Fast Attitude Maneuver Technology Based on Flywheel and Thruster Joint Control, Harbin Institute of Technology, Harbin, China, 2012.

[26] L. Rui and Z. Jun, "Pseudo-inverse secondary optimization scheme for continuous thrust distribution of non-towed spacecraft," Journal of Northwestern Polytechnical University, vol. 35, no. 6, pp. 948-952, 2017.

[27] P. A. Servidia and R. S. Pena, "Spacecraft thruster control allocation problems," IEEE Transactions on Automatic Control, vol. 50, no. 2, pp. 245-249, 2005.

[28] S. Jeb, "High-efficiency thrust vector control allocation," Journal of Guidance, Control and Dynamics, vol. 37, no. 2, pp. 374-328, 2014.

[29] D. Mark and R. Pallb, "GOCE data, modelsand applications: a review," International Journal of Applied Earth Observation and Geoinformation, vol. 35, no. 1, pp. 4-15, 2015.

[30] E. Ganuto, "Drag-free and attitude control for the GOCE satellite," Automatica, vol. 44, no. 3, pp. 1766-1780, 2008. 